\title{
A novel method of cell culture based on the microfluidic chip for regulation of cell density
}

\author{
Fei Zhang ${ }^{1}$, Rongbiao Zhang ${ }^{1}$, Mingji $\mathrm{Wei}^{1}$, and Yecheng Zhang ${ }^{1}$ \\ ${ }^{1}$ Jiangsu University
}

July 29, 2020

\begin{abstract}
The regulation of cell density is an important segment in microfluidic cell culture, particularly in the repeated assays. Traditionally, the consistent cell density is difficult to achieve owing to the inaccurate regulation of cell density with manual feedback. A novel microfluidic culture method with automatic feedback is proposed for real-time regulation of cell density in this paper. Here, an integrated microfluidic system combining cell culture, density detection and control of proliferation rate was developed. Interdigital electrode structures (IDES) are sputtered on the microchannel for automatically providing the real-time feedback information of impedance. The most sensitive frequency is studied to improve the detection resolution of the sensing chip. Cells were cultured on the chip surface and the cell density was detected by monitoring the alternation of the impedance. The feedback controller is established by least squares support vector machines (LS-SVM). Then, the cell proliferation rate was automatically controlled using the feedback controller to achieve the desired cell density in the repeated assays. The results show that the standard error of this method is $2.8 \%$ indicating that the method can keep consistency of cell density in the repeated assays. This study provides a basis for improving the accuracy and repeatability in the further assays of finding the optimal drug concentration.
\end{abstract}

\section{Hosted file}

manuscript.docx available at https://authorea.com/users/347155/articles/472950-a-novelmethod-of-cell-culture-based-on-the-microfluidic-chip-for-regulation-of-cell-density 


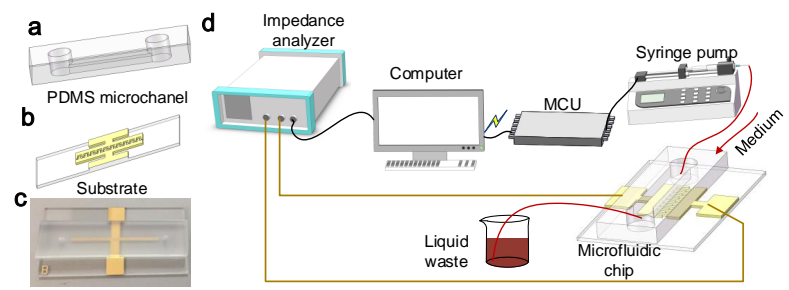

FIGURE. 1. The process of the cell culture assay. (a) The PDMS slab of the designed microfluidic chip. (b) The substrate of the microfluidic chip. The substrate is sputtered by the interdigital electrode structures. (c) The image of designed microfluidic chip. It is composed of PDMS micro-channel and substrate (d) The experiment setup of the cell culture incorporated with impedance measurement. Springe pump is connected with MCU to control the flow rate.
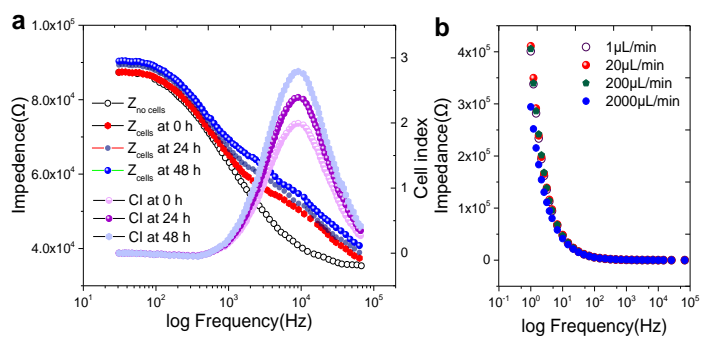

C
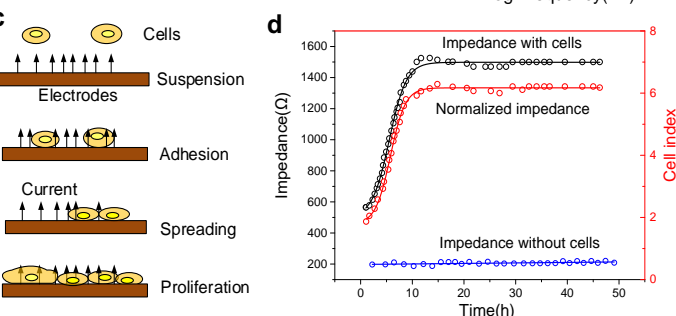

FIGURE. 2. The relationship between impedance and cell proliferation (a) The sensitive frequency decided by the impedance spectroscopy. (b) The influence of flow rate on impedance without cells. (c) The schematic of cell growth on the electrodes. Cells grow from suspension to adhere, and then they are proliferated. (d) The impedance measured at $10 \mathrm{kHz}$ by the impedance analyzer. The control group is $Z_{\text {cell-free }}$ meaning impedance without cells. Cell index is described as the normalized impedance to accurate calculation and eliminates the effect during the cell 